\title{
MANISFESTAÇÃO PRIMÁRIA DE SÍFILIS EM CAVIDADE ORAL
}

\author{
PRIMARY MANIFESTATION OF SYPHILIS IN ORAL CAVITY
}

\section{G ACESSO LIVRE}

Citação: Soerger P, Dias PHFS, Cruz EZ, Lima SRR, Santos FP, Rosa ACG (2021) Manisfestação primária de sífilis em cavidade oral. Revista de Patologia do Tocantins, 8(2).

Instituição: ${ }^{1}$ Graduanda em Medicina Instituto Tocantinense Presidente Antônio Carlos, ITPAC, Palmas, Tocantins, Brasil ${ }^{2}$ Mestre em Estomatologia e Radiologia Instituto Tocantinense Presidente Antônio Carlos, ITPAC, Palmas, Tocantins, Brasil ${ }^{3}$ Cirurgião Buco Maxilo Facial

Centro de Especialidades Odontológicas de Palmas, Tocantins. Brasil. Hospital Geral Público de Palmas, Tocantins, Brasil. ${ }^{4}$ Doutor em Patologia Bucal. Faculdade de Odontologia São Leopoldo Mandic, Campinas, São Paulo, Brasil. ${ }^{5}$ Doutora em Patologia Bucal. Instituto Tocantinense Presidente Antônio Carlos, ITPAC, Palmas, Tocantins, Brasil. Universidade Federal do Tocantins.

Autor correspondente: Ana Cláudia Garcia Rosa. Universidade Federal do Tocantins, Curso de Medicina. Quadra 109 Norte, Avenida NS-15, ALCNO-14. Plano Diretor Norte | 77001-090 | Palmas/TO. Fone: +55 (63) 3229- $4658 . \quad$ Email: anaclaudiagarcia@uft.edu.br

Editor: Rosa A. C. G. Medicina, Universidade Federal do Tocantins, Brasil.

Publicado: 25 de julho de 2021.

Direitos Autorais: (c) 2021 Soerger et al. Este é um artigo de acesso aberto que permite o uso, a distribuição e a reprodução sem restrições em qualquer meio, desde que o autor original e a fonte sejam creditados.

Conflito de interesses: os autores declararam que não existem conflitos de interesses.

Patrícia Soerger ${ }^{1}$, Pedro Henrique Fernandes Siqueira Dias ${ }^{1}$, Eduardo Zambaldi da $\mathrm{Cruz}^{2}$, Sandro Regis Rodrigues Lima ${ }^{3}$, Fabrício Passador Santos ${ }^{4}$, Ana Cláudia Garcia Rosa ${ }^{5}$.

\begin{abstract}
RESUMO
Introdução: a sífilis é uma infecção sexualmente transmissível (IST), causada pela bactéria espiroqueta Treponema pallidum (TP). A doença pode manifestar-se de diversas formas clínicas quando adquirida (primária, secundária e terciária), e também pode ser congênita. Todas as formas de sífilis podem apresentar manifestações bucais, embora na cavidade oral as lesões sifilíticas sejam incomuns. Atualmente, tem sido observado um aumento na incidência de sífilis entre adultos jovens, especialmente entre os HIV positivos. Desenvolvimento: o objetivo desse trabalho é relatar um caso de sífilis com manifestação oral. Um paciente do sexo masculino de 26 anos, leucoderma, fumante, homossexual, compareceu a um serviço de odontologia para avaliação de lesão ulcerada no palato. Durante a anamnese, relatou ter tido sífilis e que a doença havia sido adequadamente tratada com penicilina $\mathrm{G}$ benzatina. Ao exame físico, foi constatada uma lesão única, ulcerada, de base clara e indolor, localizada isoladamente entre o palato mole e duro, na região de linha média. O paciente não apresentava lesões cutâneas. As hipóteses diagnósticas foram de carcinoma epidermoide ou sífilis. Foi realizada uma biópsia incisional. A análise histopatológica e imunohistoquímica evidenciou a presença de espiroquetas no espécime estudado, e o diagnóstico foi confirmado com exames sorológicos. Considerações finais: mesmo com grandes avanços em relação à prevenção sexual, a sífilis ainda é considerada um problema de saúde pública, especialmente pelas dificuldades em se identificar as lesões orais características, que podem manifestar-se de diversas formas. A prática de relação sexual desprotegida associada à presença de lesões orais ulceradas ou erosivas pode ser indicador de sífilis, o que deve ser bem avaliado durante uma consulta direcionada ao diagnóstico.

Palavras-Chave: sífilis; sífilis cutânea; cavidade oral; boca; sífilis primária, secundária e terciária; manifestações orais; treponema pallidum
\end{abstract}

\begin{abstract}
Introduction: syphilis is a sexually transmitted infection (STI) caused by the spirochete bacteria Treponema pallidum (TP). The disease can manifest itself in several clinical forms when acquired (primary, secondary and tertiary) and can also be congenital. All forms of syphilis can present with oral manifestations, although syphilitic lesions are uncommon in the oral cavity. Currently, an increase in the incidence of syphilis has been observed among young adults, especially HIV positive patients. Development: the objective of this work is to report a case of syphilis with oral manifestation. A 26-year-old male patient, leucoderma, smoker, homosexual, attended a dental service to assess ulcerated lesion on the palate. During the anamnesis, she reported having had syphilis and that the disease had been adequately treated with benzathine penicillin G. On physical examination, a single, ulcerated lesion was found, with a clear and painless base, located separately between the soft and hard palate, in the midline region. The patient had no skin lesions. The diagnostic hypotheses were squamous cell carcinoma or syphilis. An incisional biopsy was performed. Histopathological and immunohistochemical analysis showed the presence of spirochetes in the studied specimen, and the diagnosis was confirmed with serological tests. Final considerations: even with great advances in relation to sexual prevention, syphilis is still considered a public health problem, especially due to the difficulties in identifying the characteristic oral lesions, which can manifest themselves in different ways. The practice of unprotected sexual intercourse associated with the presence of ulcerated or erosive oral lesions can be an indicator of syphilis, which should be well evaluated during a consultation directed at diagnosis.
\end{abstract}

Key words: syphilis; cutaneous syphilis; oral cavity; mouth; primary, secondary and tertiary syphilis; oral manifestations; treponema pallidum 


\section{INTRODUÇÃO}

A sífilis, ou lues, é uma infecção crônica bacteriana causada pela bactéria espiroqueta Treponema pallidum. A teoria mais aceita diz que essa doença foi levada das Américas para Europa, pelo navegador Italiano Cristóvão Colombo por volta de $1492^{1}$. Outros acreditam que Hipócrates tenha documentado casos da enfermidade na Grécia Antiga em meados de 600 A.C ${ }^{1}$. Clinicamente, o alemão Ulrich von Hutten foi a primeira representação genuína de um paciente afetado pela forma crônica da doença².

As principais formas de transmissão da sífilis são por meio de relações sexuais a partir do contato com lesão cutânea ou de mucosa do parceiro sexual infectado e por via placentária (congênita). Pode ser também adquirida através de transfusão sanguínea ${ }^{3}$. Quando adquirida, a doença manifesta-se clinicamente de diversas formas, incluindo apresentações orais em todos os estágios (primário, secundário e terciário), sendo mais comum a manifestação oral na fase secundária ${ }^{4}$.

A sífilis primaria manifesta-se através do cancro, o qual se torna visível após 3 a 90 dias da inoculação, e se desenvolve como uma lesão papular endurecida e evolui como uma úlcera central indolor com margens bem definidas, caracterizado por uma base úmida e "limpa" 3. A fase secundária da sífilis se apresenta clinicamente entre a 4 ạ e a 10 ạ semana após a infecção pelo $T$. pallidum com a presença de sintomas sistêmicos, como linfadenopatia indolor, dor de garganta, cefaleia, dor muscular, perda de peso e febre. Um de seus sinais característicos é uma erupção cutânea maculopapular difusa e indolor, espalhada por diversas regiões até mesmo a palmoplantar, podendo envolver também a cavidade oral ${ }^{3}$. Na sífilis terciaria, após um período de latência que pode durar de 1 a 30 anos podem manifestar-se complicações mais serias como: desordens cardiovasculares consequentes de um arterite prévia, tais como aneurisma da aorta descendente, hipertrofia ventricular esquerda, regurgitação aórtica e insuficiência cardíaca congestiva. Nesse estágio o sistema nervoso centra (SNC) também pode ser afetado levando a tabes dorsalis, paralisia generalizada, psicose, demência, paresia e morte. Lesões oculares também podem ocorrer ${ }^{3}$.

Estima-se que existam mais de 12 milhões de casos por ano no mundo, dos quais 900 mil estão no Brasil ${ }^{5}$. No Brasil, a população mais afetada pela sífilis são as mulheres, principalmente as negras e jovens, na faixa etária de 20 a 29 anos $^{5}$. Por ser um agravo de notificação compulsória desde 2010, a sífilis teve sua taxa de detecção aumentada de 2,0 casos por 100 mil habitantes em 2010 para 58,1 casos por 100 mil habitantes em 2017. Entre 2016 e 2017, verificou-se que houve crescimento nas taxas de detecção de sífilis adquirida, especialmente na Região Norte (de 22,9 para 33,2 casos por 100 mil habitantes; $45 \%)^{5}$. O estado do Tocantins está entre os sete estados com maior detecção (70,3 casos/100 mil hab. $)^{5}$.

Um ressurgimento de um aumento do número de casos de sífilis com manifestações orais, notadamente em homens que se relacionam sexualmente com homens tem sido observado nos últimos anos. A propagação da doença pode estar diretamente associada com a prática de sexo oral por essa população, com maior incidência em jovens de 25 a 29 anos (88\% dos casos) $)^{6,7}$. Associado a isso, esse comportamento de risco traz preocupações para um aumento paralelo na transmissão do HIV, onde uma coinfecção pode ser capaz de mudar as formas de manifestações da sífilis com um maior acometimento de órgãos, erupções cutâneas atípicas e uma progressão mais rápida para neurosífilis ${ }^{6,7}$.

O objetivo desse trabalho é relatar um caso de sífilis com manifestação oral em um homem homossexual, imunocompetente, de 26 anos de idade.

\section{DESCRIÇÃO DO CASO}

Este trabalho foi aprovado pelo Comitê de Ética em Pesquisa da UNITPAC, sob número CAAE 99151718.9.0000.0014. Um paciente do sexo masculino de 26 anos, leucoderma, fumante, homossexual, compareceu a um serviço de odontologia para avaliação de lesão ulcerada no palato. Durante a anamnese, relatou ter tido sífilis e que a doença havia sido adequadamente tratada com penicilina $G$ benzatina. Ao exame físico, foi constatada uma lesão única, ulcerada, de base clara e indolor, localizada isoladamente entre o palato mole e duro, na região de linha média (Fig.1A).

\section{Figura 1}

Figura 1 - A: aspecto clínico da lesão. B - D: aspectos histopatológicos (HE 40X, 100X e 200x, respectivamente). E-F: expressão imuno-istoquímica do anticorpo anti - Treponema pallidum evidenciando a presença de espiroquetas no tecido inflamatório.

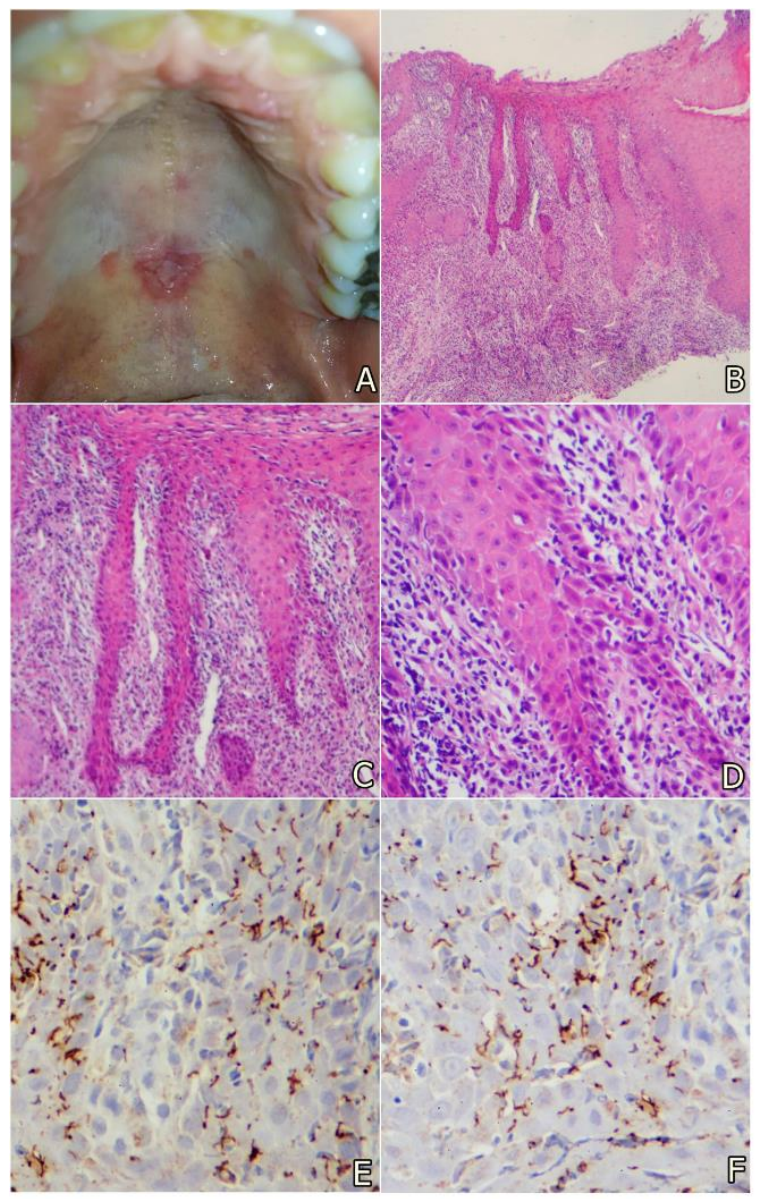


O paciente não apresentava lesões cutâneas. As hipóteses diagnósticas foram de carcinoma epidermoide ou sífilis. Foi realizada uma biópsia incisional. A análise histopatológica mostrou um epitélio estratificado pavimentoso paraqueratinizado com hiperplasia pseudoepiteliomatosa, ulceração, acantose e exocitose acentuada, além de um intenso infiltrado inflamatório crônico composto por plasmócitos e linfócitos no tecido conjuntivo (Fig.1B, 1C e 1D). A área ulcerada encontrava-se parcialmente recoberta por membrana fibropurulenta. A combinação de intensa exocitose e denso infiltrado linfoplasmocitário subjacente sugeriu o diagnóstico de sífilis, o que justificou a procura pelo micro-organismo. Reações imuno-histoquímicas para a bactéria (anticorpo antiTreponema pallidum) identificaram um grande número de espiroquetas no espécime estudado (Fig.1E e 1F). O diagnóstico de sífilis foi então confirmado pelos testes complementares VDRL e FTA-ABS, e o paciente foi tratado com eritromicina por apresentar reação alérgica à penicilina, sem recidiva após dois anos de seguimento.

\section{DISCUSSÃO}

A manifestação de sífilis em cavidade oral foi descrita pela primeira vez em 1955, por Huebsch ${ }^{8}$. Em uma análise de 66 casos encontrados na literatura em língua inglesa até a presente data (Tabela 1), verifica-se que o acometimento da cavidade oral é predominante em pacientes do sexo masculino, com idade entre 35 a 40 anos. As lesões apresentam-se mais sob a forma ulcerada ou sob a forma de manchas mucosas (Tabela 1).

Tabela 1

Casos de sífilis com manifestação oral previamente relatados na literatura (1955-2019). Sexo masculino (M) ou feminino (F); estágio primário (1), secundário (2) ou terciário (3).

\begin{tabular}{|c|c|c|c|c|c|c|c|}
\hline Caso & Sexo & Idade & Localização & Estágio & Aspecto clínico & $\begin{array}{c}\text { Tratame } \\
\text { nto }\end{array}$ & Referência \\
\hline 1 & M & 33 & Palato duro & 3 & Sequestro & N/A & Huebsch $(1955)^{\mathrm{g}}$ \\
\hline 2 & M & 43 & Palato duro & 3 & Ulcerações & Penicilina & $\begin{array}{c}\text { Taylor \& } \\
\text { Hipple (1961) }\end{array}$ \\
\hline 3 & M & 63 & Palato & 3 & Fenda & Penicilina & $\begin{array}{c}\text { Ramstadi \& } \\
\text { Traaholt (1980) } 10\end{array}$ \\
\hline 4 & M & 45 & $\begin{array}{c}\text { Mucosa labial } \\
\text { e Lingua }\end{array}$ & 2 & Manchas mucosas & N/A & $\operatorname{Mani}(1984)^{11}$ \\
\hline 5 & M & 28 & Mucosa labial & 2 & Manchas mucosas & N/A & $\operatorname{Mani}(1984)^{11}$ \\
\hline 6 & $\mathrm{~F}$ & 19 & $\begin{array}{c}\text { Mucosa labial } \\
\text { e bucal }\end{array}$ & 2 & Manchas mucosas & $\mathrm{N} / \mathrm{A}$ & $\operatorname{Mani}(1984)^{11}$ \\
\hline 7 & M & 35 & Gengiva & 2 & $\begin{array}{c}\text { Ulcerações } \\
\text { coalescentes } \\
\text { múltiplas }\end{array}$ & Penicilina & Ficarra et al (1993) 12 \\
\hline 8 & M & 31 & Palato duro & 2 & Manchas mucosas & Penicilina & Ban et al $(1995)^{13}$ \\
\hline 9 & F & 38 & $\begin{array}{c}\text { Mucosa labial } \\
\text { e Língua }\end{array}$ & 2 & $\begin{array}{c}\text { Lesões } \\
\text { superficiais } \\
\text { ulceradas com } \\
\text { pseudomembranas }\end{array}$ & Penicilina & $\begin{array}{l}\text { Junkins-Hopkins \& Hood } \\
\qquad(1996)^{14}\end{array}$ \\
\hline 10 & F & 6 & Língua & 2 & Manchas mucosas & Penicilina & Oztürk et al (1998) is \\
\hline 11 & M & 31 & Palato & 1 & Ulceração & Penicilina & Alam et al $(2000)^{16}$ \\
\hline 12 & M & 61 & Mucosa labial & 1 & Ulceração & Penicilina & Alam et al $(2000)^{16}$ \\
\hline 13 & M & 35 & $\begin{array}{c}\text { Palato, } \\
\text { mucosa bucal }\end{array}$ & 2 & Manchas mucosas & Penicilina & Lu \& Eng (2002) ${ }^{17}$ \\
\hline 14 & F & 38 & Palato & 2 & Manchas mucosas & Penicilina & Lu \& Eng (2002) ${ }^{17}$ \\
\hline 15 & M & 30 & Mucosa bucal & 2 & Manchas mucosas & Penicilina & Lu \& Eng (2002) 17 \\
\hline 16 & M & 58 & Mucosa labial & 2 & Ulceração & Penicilina & Lu \& Eng (2002) ${ }^{1 ?}$ \\
\hline 17 & M & 41 & Língua & 2 & Tipo leucoplasia & Penicilina & Aquilina et al (2003) ${ }^{1 s}$ \\
\hline 18 & F & 24 & Comissura & 1 & Ulceração & Penicilina & Scott \& Flint (2005) ${ }^{19}$ \\
\hline 19 & M & 36 & Comigsura & 1 & Ulceração & Penicilina & Scott \& Flint $(2005)^{19}$ \\
\hline 20 & M & 28 & Mucosa labial & 1 & Ulceração & Penicilina & Scott \& Flint $(2005)^{19}$ \\
\hline 21 & M & 31 & Mucosa bucal & 1 & Ulceração & $\begin{array}{c}\text { Eritromici } \\
\text { na }\end{array}$ & Veraldi et al $(2008)^{20}$ \\
\hline 22 & M & 38 & Língua & 2 & Ulceração & $\underset{\mathbf{a}}{\text { Doxiciclin }}$ & Bruce et al $(2009)^{21}$ \\
\hline 23 & M & 58 & Palato & 2 & Ulceração & Penicilina & Murrel et al $(2009)^{21}$ \\
\hline 24 & M & 52 & Língua & 1 & Ulcerações & Penicilina & Shumway et al (2009) ${ }^{23}$ \\
\hline 25 & $\mathrm{M}$ & 45 & Líng̨ua & 1 & Ulceração & $\mathrm{N} / \mathrm{A}$ & Ramoni et al (2009) ${ }^{24}$ \\
\hline 26 & M & 37 & $\begin{array}{l}\text { Fómice do } \\
\text { vestibulo }\end{array}$ & 1 & Ulceração & N/A & Ramoni et al (2009) ${ }^{24}$ \\
\hline 27 & M & 28 & Língua & 1 & Ulceração & Penicilina & Ramoni et al (2009) ${ }^{24}$ \\
\hline 28 & M & 45 & $\begin{array}{c}\text { Mucosa bucal } \\
\text { e lingua }\end{array}$ & 2 & Tipo leucoplasia & $\begin{array}{c}\text { Eritromici } \\
\text { na }\end{array}$ & Compilato et al $(2009)^{25}$ \\
\hline 29 & F & 24 & Mucosa bucal & 2 & Ulceras aftosas & $\begin{array}{c}\text { Doxiciclin } \\
\mathbf{a}\end{array}$ & Ibrahim \& Malu (2009) ${ }^{26}$ \\
\hline 30 & M & 37 & Lingua & 2 & Ulceração & $\underset{\mathbf{a}}{\text { Doxiciclin }}$ & Flynn et al $(2010)^{27}$ \\
\hline 31 & M & 67 & Lingua & 2 & Ulceração & N/A & Ikenberg et al $(2010)^{28}$ \\
\hline 32 & M & 47 & Língua & 2 & Ulceração & $\mathrm{N} / \mathrm{A}$ & Ikenberg et al $(2010)^{2 \mathrm{R}}$ \\
\hline 33 & M & 39 & Mucosa labial & 2 & Ulceração & Penicilina & Rajlawat et al $(2011)^{29}$ \\
\hline 34 & M & 41 & Língua & 1 & Ulceração & Penicilina & Staines \& Sloan (2011) ${ }^{30}$ \\
\hline 35 & M & 25 & $\begin{array}{l}\text { Tonsilas } \\
\text { palatinas }\end{array}$ & 2 & Ulcerações & Penicilina & Gedela \& Boag (2012) 31 \\
\hline 36 & M & 79 & $\begin{array}{l}\text { Palato duro e } \\
\text { mole }\end{array}$ & 2 & $\begin{array}{c}\text { Lesão } \\
\text { maculopapular }\end{array}$ & Penicilina & Minicucci et al (2013) ${ }^{12}$ \\
\hline 37 & M & 43 & $\begin{array}{c}\text { Palato e } \\
\text { frênulo labial }\end{array}$ & 2 & Ulcerações & Penicilina & Hertel et al $(2014)^{13}$ \\
\hline 38 & F & 33 & $\begin{array}{l}\text { Lábios, palato } \\
\text { duro, mucosa } \\
\text { bucal } \\
\text { esquerda, } \\
\text { ponta da } \\
\text { lingua e } \\
\text { comisguras }\end{array}$ & 2 & Lesão vesicular & Penicilina & Hertel et al $(2014)^{33}$ \\
\hline 39 & $\mathrm{M}$ & 42 & $\begin{array}{c}\text { Comiggura } \\
\text { direita }\end{array}$ & 2 & Ulcerações & Penicilina & Hertel et al $(2014)^{33}$ \\
\hline 40 & $\mathrm{M}$ & 43 & $\begin{array}{l}\text { Palato duro, } \\
\text { labios, face } \\
\text { vestibular } \\
\text { esquerda da } \\
\end{array}$ & 2 & Manchas mucosas & Penicilina & Hertel et al (2014) ${ }^{33}$ \\
\hline
\end{tabular}




\begin{tabular}{|c|c|c|c|c|c|c|c|}
\hline & & & $\begin{array}{c}\text { mucosa e } \\
\text { ponta da } \\
\text { lingua }\end{array}$ & & & & \\
\hline 41 & F & 60 & $\begin{array}{c}\text { Ponta da } \\
\text { lingua e } \\
\text { bilateralmente } \\
\text { na mucosa } \\
\text { bucal } \\
\end{array}$ & 2 ou $3 ?$ & $\begin{array}{c}\text { Pápulas } \\
\text { eritematosas }\end{array}$ & $\begin{array}{c}\text { Tetracicli } \\
\text { na }\end{array}$ & Hertel et al $(2014)^{33}$ \\
\hline 42 & F & 32 & Lábio superior & 1 & Ulceração & Penicilina & Vera-Kellet et al (2014) ${ }^{34}$ \\
\hline 43 & M & 35 & Língua & 2 & Ulceração & Penicilina & Safe \& Maia $(2014)^{35}$ \\
\hline 44 & M & 19 & $\begin{array}{c}\text { Palato duro, } \\
\text { labio inferior } \\
\text { e lingua }\end{array}$ & - & Manchas mucosas & Penicilina & De Paulo et al $(2015)^{36}$ \\
\hline 45 & $\mathrm{~F}$ & 40 & $\begin{array}{l}\text { Lábio inferior } \\
\text { e superior }\end{array}$ & - & Manchas mucosas & Penicilina & De Paulo et al $(2015)^{36}$ \\
\hline 46 & F & 25 & $\begin{array}{c}\text { Mucosa oral e } \\
\text { lingua }\end{array}$ & - & Manchas mucosas & Penicilina & De Paulo et al $(2015)^{36}$ \\
\hline 47 & $\mathrm{M}$ & 32 & $\begin{array}{l}\text { Mucosa orale } \\
\text { lábio superior }\end{array}$ & - & Manchas mucosas & Penicilina & De Paulo et al $(2015)^{36}$ \\
\hline 48 & F & 9 & $\begin{array}{l}\text { Lábio superior } \\
\text { e lingua }\end{array}$ & - & Manchas mucosas & Penicilina & De Paulo et al $(2015)^{36}$ \\
\hline 49 & M & 29 & $\begin{array}{c}\text { Mucosa oral e } \\
\text { lingua }\end{array}$ & - & Tipo leucoplasia & Penicilina & De Paulo et al $(2015)^{36}$ \\
\hline 50 & F & 21 & $\begin{array}{l}\text { Palato duro e } \\
\text { labio inferior }\end{array}$ & - & Tipo leucoplasia & Penicilina & De Paulo et al $(2015)^{36}$ \\
\hline 51 & M & 45 & $\begin{array}{c}\text { Lábio } \\
\text { superior, } \\
\text { ponta da } \\
\text { lingua e } \\
\text { comisgura } \\
\text { labial } \\
\end{array}$ & 2 & $\begin{array}{l}\text { Ulceração, placas } \\
\text { mucosas e } \\
\text { nodulaçôes }\end{array}$ & Penicilina & Seibt \& Mumerato (2016) ${ }^{37}$ \\
\hline 52 & $\mathrm{M}$ & 46 & $\begin{array}{l}\text { Palato duro, } \\
\text { mucosa do } \\
\text { labio inferior } \\
\text { e lingua }\end{array}$ & 2 & $\begin{array}{c}\text { Ulceraçäo e } \\
\text { placas mucosas }\end{array}$ & Penicilina & Seibt \& Munerato (2016) ${ }^{37}$ \\
\hline 53 & M & 42 & $\begin{array}{c}\text { Bordas da } \\
\text { lingua } \\
\text { bilateralmente }\end{array}$ & 2 & Nodulações & Penicilina & Seibt \& Munerato (2016) ${ }^{37}$ \\
\hline 54 & F & 53 & $\begin{array}{l}\text { Palato mole e } \\
\text { tonsilas }\end{array}$ & 2 & $\begin{array}{l}\text { Ulcerações e } \\
\text { nodulações }\end{array}$ & Penicilina & Seibt \& Munerato (2016) ${ }^{37}$ \\
\hline 55 & F & 29 & $\begin{array}{l}\text { Palato mole e } \\
\text { duro, mucosa } \\
\text { do labio } \\
\text { inferior } \\
\end{array}$ & 2 & $\begin{array}{c}\text { Manchas mucosas } \\
\text { e erosóes }\end{array}$ & Penicilina & Seibt \& Munerato $(2016)^{37}$ \\
\hline 56 & F & 39 & $\begin{array}{c}\text { Apice e borda } \\
\text { da lingua } \\
\text { bilateralmente }\end{array}$ & 2 & Placas mucosas & Penicilina & Seibt \& Munerato (2016) ${ }^{37}$ \\
\hline 57 & F & 36 & $\begin{array}{c}\text { Uvula e } \\
\text { tonsila } \\
\text { palatina }\end{array}$ & 2 & Manchas mucosas & Penicilina & Seibt \& Munerato (2016) ${ }^{37}$ \\
\hline 58 & M & 53 & $\begin{array}{c}\text { Palato mole e } \\
\text { mucosa oral } \\
\text { esquerda }\end{array}$ & - & Ulcerações & Penicilina & Dybeck Udd \& Lund (2016) ${ }^{3 \mathrm{~B}}$ \\
\hline 59 & M & 36 & $\begin{array}{c}\text { Apice da } \\
\text { lingua }\end{array}$ & $1 / 2$ & Ulcerações & Penicilina & Fregnamilet al (2016) ${ }^{39}$ \\
\hline 60 & M & 30 & $\begin{array}{l}\text { Mucosa } \\
\text { orolabial }\end{array}$ & - & Ulcerações & Penicilina & Onetti et al $(2017)^{40}$ \\
\hline 61 & F & 20 & $\begin{array}{l}\text { Lingua e } \\
\text { lábios }\end{array}$ & 2 & Ulcerações & Penicilina & De Andrade et al (2018) ${ }^{41}$ \\
\hline 62 & F & 42 & $\begin{array}{c}\text { Comigsura } \\
\text { labial, lingua } \\
\text { e labios }\end{array}$ & 2 & Ulcerações & Penicilina & De Andrade et al (2018) ${ }^{41}$ \\
\hline 63 & F & 17 & $\begin{array}{c}\text { Lábio, palato } \\
\text { mole e } \\
\text { mucosa oral } \\
\end{array}$ & 2 & Ulcerações & Penicilina & De Andrade et al (2018) ${ }^{41}$ \\
\hline 64 & M & 28 & $\begin{array}{c}\text { Comigsura } \\
\text { labial }\end{array}$ & 2 & Ulcerações & Penicilina & De Andrade et al $(2018)^{41}$ \\
\hline 65 & M & 59 & Palato & 1 & Ulceração & Penicilina & Streight et al (2019) ${ }^{42}$ \\
\hline 66 & F & 49 & Língua & 2 & Manchas mucosas & Penicilina & Mari et al (2019) \\
\hline
\end{tabular}

Quanto à localização, as lesões ocorrem principalmente na língua e palato, podendo acometer outras regiões, como: mucosas labiais e bucais, além da gengiva e tonsilas, que apresentam menor acometimento. $O$ segundo estágio da sífilis é a fase predominante em que ocorrem as lesões orais características (Tabela 1). No nosso caso, a doença foi diagnosticada por meio de uma ulceração no palato em um homem homossexual de 26 anos de idade. Comparando com os dados existentes (Tabela 1), observa-se que as características das manifestações orais de sífilis previamente descritas assemelham-se à maioria das características observadas no nosso paciente.

Curiosamente, um acentuado aumento no número de casos de sífilis com manifestações orais vem sendo relatado nas últimas duas décadas em comparativo com o mesmo período anterior, acometendo especialmente homens que praticam relações com pessoas do mesmo sexo e também HIV positivos ${ }^{6}$. A principal hipótese para corroborar com esses dados é um possível aumento na prática de relações sexuais orais desprotegidas, pois o fato de a transmissão do vírus HIV ser mínima por essa via faz com que medidas de prevenção sejam menos utilizadas. Contudo, não há relação direta do vírus HIV com a sífilis, apenas relatos de que a imunossupressão predispõe o indivíduo a desenvolver ou evoluir a doença bacteriana, além de dificultar o tratamento com antibióticos. Os achados histopatológicos das lesões orais causadas pela sífilis não são específicos, ocorrendo uma variação entre as fases. Na sífilis primária e secundária, o padrão é semelhante, onde observa-se uma superfície epitelial ulcerada ${ }^{4}$. No caso das lesões da segunda fase, podem ser tanto ulceradas como hiperplásicas, com significativa espongiose e exocitose ${ }^{4}$. A lâmina própria (tecido conjuntivo) subjacente se apresenta uma intensa reação inflamatória crônica, predominantemente composta por linfócitos e plasmócitos com um aumento do número de canais vasculares ${ }^{4}$. Nas lesões orais de terceira fase, o padrão histológico exibe uma superfície hiperplásica pseudoepiteliomatosa periférica. 0 infiltrado inflamatório subjacente é granulomatoso com a presença de histiócitos e células gigantes multinucleadas ${ }^{4}$. A multiplicidade de achados histológicos na sífilis está de acordo com o amplo espectro de apresentações clínicas da doença em seus diferentes estágios. A imuno-istoquímica pode ser um método muito sensível na detecção do Treponema pallidum (Fig.1E e 1F). Esta técnica é de particular interesse nos casos em que a possibilidade de diagnóstico de sífilis foi não considerada clinicamente, sendo de grande valia para o diagnóstico final.

Em relação aos exames sorológicos, existem duas categorias de testes para o diagnóstico de sífilis: exames diretos e testes imunológicos, sendo o último o mais utilizado na prática clínica, e são divididos em treponêmicos e não treponêmicos ${ }^{44}$. Os treponêmicos são testes que detectam anticorpos específicos para antígenos de $T$. pallidum e seu principal representante é o FTA-ABS, já os não treponemicos são testes não específicos contra o T. pallidum, detectando anticorpos anticardiolipínicos, reagínicos ou lipoídicos, sendo o VDRL e o Teste Rápido (TR) os mais comumente utilizados ${ }^{44}$. Em nosso caso, o paciente foi submetido à biópsia incisional da lesão oral, com análise histopatológica e visualização dos treponemas por meio de reações imuno-istoquímica que identificaram a presença das bactérias no espécime estudado. Posteriormente, a confirmação diagnóstica da doença também foi realizada por meio dos exames sorológicos VDRL e FTA-ABS. Quanto ao tratamento da doença, a penicilina tem sido o método mais empregado na maioria dos casos, desde que a efetividade dessa droga sobre o treponema foi descoberta por Mahoney em $1943{ }^{45}$. Observa-se eficácia no uso da penicilina para a regressão de lesões sifilíticas em fases primária e secundária com a aplicação de apenas uma dose (2.400.000 UI). A penicilina permanece sendo a droga de escolha até os dias atuais, embora alguns casos de sífilis sejam tratados com doxiciclina, ceftriaxona ou eritromicina, que apresentam menor eficácia, mas são indicados para pacientes comprovadamente alérgicos a penicilina ${ }^{44}$. Nestes casos, o Ministério da Saúde recomenda tratamento com eritromicina (na forma estearato ou estolato) $500 \mathrm{mg}$, via oral, de 6 em 6 horas, por 15 dias para sífilis recente e por 30 dias para sífilis tardia; ou com tetraciclina, na mesma dose; ou doxiciclina 100mg, por via oral, de 12 em 12 horas, por 15 dias na sífilis recente e por 30 dias na sífilis tardia. A tetraciclina, a doxiciclina 
e o estolato de eritromicina são contraindicados na gestação ${ }^{5}$. No nosso caso, o paciente foi tratado com eritromicina, sem apresentar recidiva das lesões orais após dois anos de seguimento.

\section{CONCLUSÃO}

Mesmo com grandes avanços em relação à prevenção sexual, a sífilis ainda é considerada um problema de saúde pública, especialmente pelas dificuldades em se identificar as lesões orais características, que podem manifestar-se de diversas formas. A prática de relação sexual desprotegida associada à presença de lesões orais ulceradas ou erosivas pode ser indicador de sífilis, o que deve ser bem avaliado durante uma consulta direcionada ao diagnóstico.

O tratamento da sífilis deve ser feito por uma equipe multidisciplinar, que acompanhe o paciente durante um período adequado de tempo. Nesse âmbito, os profissionais de saúde possuem um papel importante na identificação da sífilis oral, para que o paciente possa receber um tratamento adequado, possibilitando um bom prognóstico com o mínimo de complicações.

\section{REFERÊNCIAS BIBLIOGRÁFICAS}

1. 1- Schuch L, da Silva K, de Arruda J, Etges A, Gomes A, Mesquita $R$, et al. Forty cases of acquired oral syphilis and a review of the literature. International Journal of Oral and Maxillofacial Surgery. 2019;48(5):635-43.

2. 2- Riccomi G, Giuffra V. First Portrait of a Syphilitic Patient: Ulrich von Hutten. The American journal of medicine. 2018;131(6):714-5.

3. 3- Abbas AK, Fausto N, Kumar V, Mitchell RN. Robbins Patologia Básica9: Robbins Patologia Básica: Elsevier Health Sciences.

4. 4- Neville B. Patologia oral e maxilofacial: Elsevier Brasil; 2011.

5. 5- Saúde SdVe, Saúde Md. Boletim epidemiológico: sífilis. Departamento de Vigilância, Prevenção e Controle das IST, do HIV/Aids e das ...; 2017.

6. 6- Thompson LD. Oral Syphilis. Ear, Nose \& Throat Journal. 2019:0145561319890154.

7. 7- Ramírez-Amador V, Anaya-Saavedra G, Crabtree-Ramírez B, Esquivel-Pedraza L, Saeb-Lima M, Sierra-Madero J. Clinical spectrum of oral secondary syphilis in HIV-infected patients. Journal of Sexually Transmitted Diseases. 2013;2013.

8. 8- Huebsch RF. Gumma of the hard palate, with perforation; report of a case. Oral Surg Oral Med Oral Pathol. 1955 Jul;8(7):690-3.

9. 9- Taylor RG, Hipple W. Gumma of palate with negative standard tests for syphilis. Oral Surgery, Oral Medicine, Oral Pathology. 1961;14(7):788-92.
10. 10- Ramstad T, Traaholt L. Destruction of the soft palate and nose by tertiary'benign'syphilis. A case report. Journal of oral rehabilitation. 1980;7(2):111-5

11. 11- Mani N. Secondary syphilis initially diagnosed from oral lesions: Report of three cases. Oral surgery, oral medicine, oral pathology. 1984;58(1):47-50.

12. 12- Ficarra G, Zaragoza AM, Stendardi L, Parri F, Cockerell CJ. Early oral presentation of lues maligna in a patient with HIV infection. A case report. Oral Surg Oral Med Oral Pathol. 1993;75(6):728-32.

13. 13- Ban M, Ohtani $M$, Seishima M. A case of secondary syphilis with mucous patches on the hard palate. The Journal of Dermatology. 1995;22(1):52-4.

14. 14- Junkins-Hopkins JM, Hood AF. Multiple painful oral ulcerations. Archives of family medicine. 1996;5(7):379.

15. 15- Oztürk F, Gürses N, Sancak R, Bay A, Baris S. Acquired secondary syphilis in a 6-year-old girl with no history of sexual abuse. Cutis. 1998 Sep;62(3):150-1.

16. 16- Alam F, Argiriadou A, Hodgson T, Kumar N, Porter S. Primary syphilis remains a cause of oral ulceration. British Dental Journal. 2000;189(7):352-4.

17. 17- Lu S-Y, Eng H-L. Secondary syphilis-related oral ulcers: report of four cases. Chang Gung Medical Journal. 2002;25(10):683-8.

18. 18- Aquilina $C$, Viraben $R$, Denis $P$. Secondary syphilis simulating oral hairy leukoplakia. Journal of the American Academy of Dermatology. 2003;49(4):749-51.

19. 19- Scott C, Flint S. Oral syphilis-re-emergence of an old disease with oral manifestations. International journal of Oral and Maxillofacial Surgery. 2005;34(1):58-63.

20. 20- Veraldi S, Lunardon L, Persico M, Francia C, Bottini S. Multiple aphthoid syphilitic chancres of the oral cavity. Internatonal journal of STD \& AIDS. 2008;19(7):486-7.

21. 21- Bruce IA, Roper AJ, Gayed S, Dabrowski M, Morar P. Syphilitic cervical lymphadenopathy: return of an old foe. American Journal of Otolaryngology. 2009;30(5):347-9.

22. 22- Murrell GL. Secondary syphilis oral ulcer. Otolaryngology-Head and Neck Surgery. 2009;140(6):942-3.

23. 23- Shumway BS, Islam NM, Kapoor R, Huang AK, Arnold FW. Clinico-pathologic conference: case 3 . Head and neck pathology. 2009;3(4):286.

24. 24- Ramoni S, Cusini M, Gaiani F, Crosti C. Syphilitic chancres of the mouth: three cases. Acta dermato-venereologica. 2009;89(6):648-9.

25. 25-Compilato D, Amato S, Campisi G. Resurgence of syphilis: a diagnosis based on unusual oral mucosa lesions. Oral Surgery, Oral Medicine, Oral Pathology, Oral Radiology, and Endodontology. 2009;108(3):e45-e9.

26. 26- Ibrahim F, Malu M. Sudden deafness in a patient with secondary syphilis. The Journal of laryngology and otology. 2009;123(11):1262 
27. 27- Flynn TR, Hunter GJ, Johnson MM. Case 6-2010: A 37Year-Old Man with a Lesion on the Tongue. New England Journal of Medicine. 2010;362(8):740-8.

28. 28- Ikenberg K, Springer E, Bräuninger W, Kerl K, Mihic $D$, Schmid $S$, et al. Oropharyngeal lesions and cervical lymphadenopathy: syphilis is a differential diagnosis that is still relevant. Journal of clinical pathology. 2010;63(8):731-6.

29. 29- Rajlawat BP, Evans-Jones J, Triantafyllou A, Varga E, Field EA. A solitary oral ulcer. Clin Exp Dermatol. 2011 Mar;36(2):217-9.

30. 30- Staines K, Sloan P. Syphilitic chancre of the tongue. New England Journal of Medicine. 2011;365(5):e11.

31. 31- Gedela K, Boag F. Syphilitic tonsillitis in primary care: a case report. Br J Gen Pract. 2012;62(597):219-20.

32. 32- Minicucci EM, Vieira RA, Oliveira DT, Marques SA. Oral manifestations of secondary syphilis in the elderly - a timely reminder for dentists. Aust Dent J. 2013 Sep;58(3):368-70

33. 33- Hertel M, Matter D, Schmidt-Westhausen AM, Bornstein MM. Oral syphilis: a series of 5 cases. Journal of oral and maxillofacial surgery. 2014;72(2):338-45.

34. 34- Vera-Kellet C, Harz-Fresno I, Manriquez J. Labial ulcer: oral manifestation of syphilis. Brazilian Journal of Infectious Diseases. 2014;18(5):570-1.

35. 35- Safe IP, Maia DCC. Secondary syphilis with oral manifestation. Brazilian Journal of Infectious Diseases. 2014;18(1):95-

36. 36- De Paulo LFB, Servato JPS, Oliveira MTF, Durighetto Jr $A F$, Zanetta-Barbosa D. Oral manifestations of secondary syphilis. International Journal of Infectious Diseases. 2015;35:40-2

37. 37- Seibt CE, Munerato MC. Secondary syphilis in the oral cavity and the role of the dental surgeon in STD prevention, diagnosis and treatment: a case series study. The Brazilian Journal of Infectious Diseases. 2016;20(4):393-8.

38. 38- Dybeck Udd S, Lund B. Oral syphilis: a reemerging infection prompting clinicians' alertness. Case reports in dentistry. 2016;2016.

39. 39- Fregnani ER, Pérez-de-Oliveira ME, Parahyba CJ, Perez D. Primary syphilis: An uncommon manifestation in the oral cavity. Journal of the Formosan Medical Association= Taiwan yi zhi. 2017;116(4):326.

40. 40- Tognetti L, Cinotti E, Tripodi S, Garosi G, Rubegni P. Unusual presentation of secondary syphilis: membranoproliferative glomerulonephritis andmucocutaneous lesions. International journal of STD \& AIDS. 2018;29(4):410-3.

41. 41- De Andrade R-S, de Freitas E-M, Rocha B-A. Oral findings in secondary syphilis. Medicina oral, patologia oral y cirugia bucal. 2018;23(2):e 138
42. 42- Streight KL, Paranal RM, Musher DM. The oral manifestations of syphilitic disease: a case report. Journal of medical case reports. 2019;13(1):227.

43. 43- Mari E, Nudo M, Palese E, Cotticelli L, Cotticelli C, Zino G, Coppola R, Di Lella FM, Arcari G, Rodio DM, Prezioso C, Pietropaolo V, Devirgiliis V, Panasiti V. Beyond appearance: An unusual manifestation of isolated oral secondary syphilis. Int J Immunopathol Pharmacol. 2019 Jan-Dec; 33:2058738419845566.

44. 44- Sumikawa E, Motta L, Inocêncio L, Ferreira L, Bazzo $M$, Franchini $M$, et al. Sífilis: Estratégias para Diagnóstico no Brasil. 2017.

45. 45- Mahoney JF, Arnold RC, Harris A. Penicillin treatment of early syphilis-a preliminary report. American Journal of Public Health and the Nations Health. 1943;33(12):1387-91. 